Onkologe 2009 · 15:7

DOI 10.1007/s00761-008-1544-5

Online publiziert: 25 . Dezember 2008

๑) Springer Medizin Verlag 2008

K. Diedrich ${ }^{1}$ H.G. Schnürch ${ }^{2} \cdot$ M. Thill ${ }^{1} \cdot$ D. Fischer ${ }^{1}$

${ }^{1}$ Klinik für Frauenheilkunde und Geburtshilfe, Universitätsklinikum Schleswig-Holstein, Lübeck

${ }^{2}$ Frauenklinik des Lukaskrankenhauses, Neuss

\title{
Tumoren von Vagina und Vulva
}

sien, in denen insbesondere die prognostisch relevanten Faktoren und die pathologische Aufarbeitung dargestellt werden. Der Diagnostik und operativen Therapie sowohl des Primärtumors als auch der Leistenlymphknoten sind ausführliche Arbeiten gewidmet. Aufgrund der Ergebnisse einer Pilotuntersuchung der Organkommission Vulva- und Vaginaltumoren der Arbeitsgemeinschaft Gynäkologische Onkologie stehen die neuen Möglichkeiten durch die Sentinel-Technik in einem gesonderten Beitrag besonders im Fokus. Auf die Möglichkeiten der Strahlentherapie sowohl in der adjuvanten Therapieplanung als auch in der Kombination mit einer Chemotherapie bei fortgeschrittenen Vulvakarzinomen wird ebenso eingegangen. Beim Vaginalkarzinom ermöglichen eine computergesteuerte Therapieplanung und differenzierte Afterloadingverfahren eine individualisierte Behandlungsstrategie. Schließlich ist der Therapie des Rezidivs eine ausführliche Arbeit gewidmet, bei der auf die Möglichkeiten der plastisch-rekonstruktiven Verfahren dezidiert eingegangen wird.

Die Früherkennung des Vulva- und Vaginalkarzinoms ist nicht nur im Rahmen der gynäkologischen Vorsorgeuntersuchung möglich. Die Behandlung ist in der überwiegenden Mehrzahl primär operativ. Insbesondere unter der Berücksichtigung neuer Therapiekonzepte ist eine enge interdisziplinäre Zusammenarbeit mit dem Nuklearmediziner erforderlich; auch die gemeinsame Betrachtung mit dem Radioonkologen und dem Der- matologen kann für individuelle Therapiestrategien Vorteile bieten.

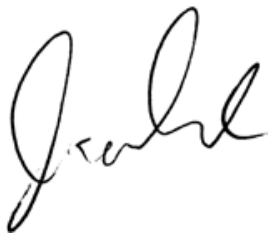

K. Diedrich

Für die Herausgeber des Schwerpunktheftes
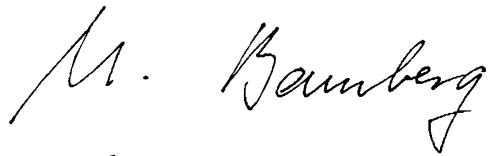

M. Bamberg

Für die Herausgeber

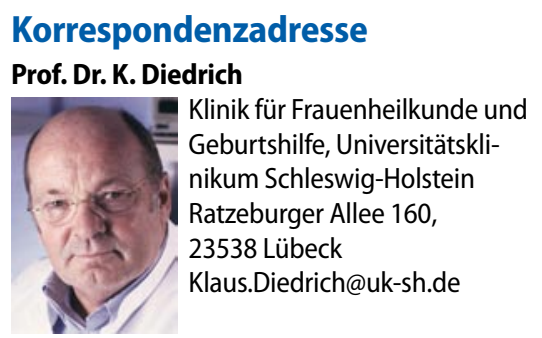

